\title{
Recent research progress in computational solid mechanics
}

\author{
ZHUANG Zhuo* \& MAITIREYIMU Maigefeireti
}

School of Aerospace, Tsinghua University, Beijing 100084, China

Received September 25, 2012; accepted November 2, 2012

\begin{abstract}
Computational mechanics has had a profound impact on science and technology over the past five decades. It has numerically transformed much of the classical theory models into practical tools for predicting and understanding the complex engineering and science system. A short review is given in this paper on some recent progress in computational solid mechanics at multi-scales.
\end{abstract}

computational solid mechanics, extended finite element method, molecule dynamic, multi-scales

Citation: Zhuang Z, Maitireyium M. Recent research progress in computational solid mechanics. Chin Sci Bull, 2012, 57: 4683-4688, doi: 10.1007/s11434$012-5580-6$

Computational mechanics has had a profound impact on science and technology over the past five decades. It has numerically transformed much of the classical Newtonian theory into practical tools for predicting and understanding the complex engineering and science system, which have some limitations using the classic analytical solutions. Finite element methodology (FEM), which is one of the most powerful computation tools, has been widely used in the simulation-based engineering and science. There are a number of methods based on FEM dealing with the problem of material behaviors and structural mechanics, for example, damage and fracture. Since the limitation of FEM is in the continuum problem ranging from micro-meter to macro scales, the molecule dynamic (MD) computation is used at nano-scale research. A short review is given in this paper on the recent progress in computational solid mechanics related to length scales.

\section{MD simulation at nano-scale}

The carbon nano-tube (CNT) as a representative nano-scale material was invented twenty years ago. Its material behavior and mechanics has been interested by many researchers and

*Corresponding author (email: zhuangz@tsinghua.edu.cn) engineers. The investigations were carried out from the aspects of theory, experiment and simulation. Since it is discrete at atomic length, the MD method is the best tool in the numerical analysis. Another purpose using MD simulation is to provide the failure criterion for dislocation initiation in the crystals, which can link the scales from nano-meter to micron. The minimum energy principle for the dislocation nucleation or initiation was predicted for inhomogeneous atomic system [1]. The simulation results illustrated that the thin film is easily nucleated on the surface because the atomic bonds lose. Based on MD simulation at nano-size and theoretical analysis, an investigation of the combined size and rate effects on the mechanical responses of faced cubic crystal (FCC) metals has been made to develop a hyper-surface (Figure 1) crossing the length scales [2].

The high strength and hard toughness properties of crystalline solid were intensively investigated at sub-micron and nanoscales. In these scales, the forms of defects in a crystal material are dislocation and grain boundary. A majority of research papers were focused on the dislocation nucleation and the interactions between dislocation and grain boundary [3]. The yield strength of perfect crystal depends on dislocation nucleation. The interactions between dislocation and grain boundary affect the plastic properties of crystal material.

Based on the atom potential method and the CauchyBorn rule, the stability theory was developed to describe the 


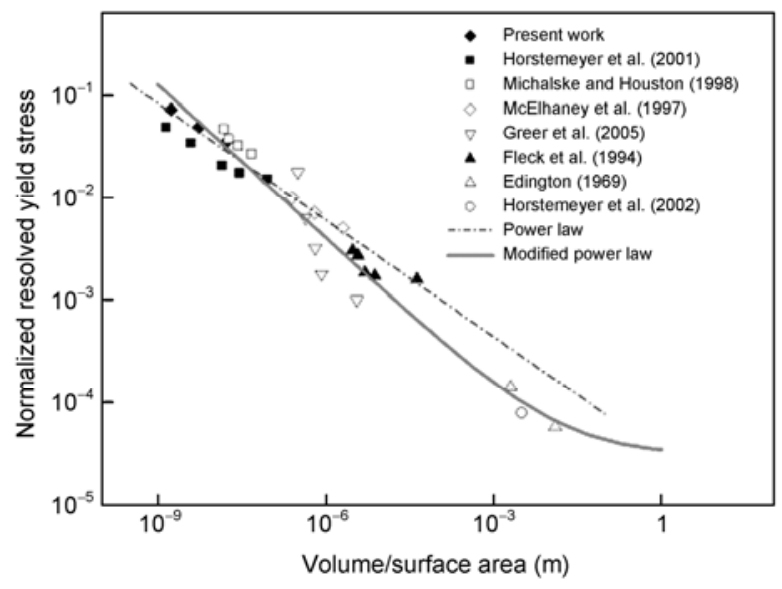

Figure 1 Yield stress normalized by the elastic modulus and resolved on a (111) slip plane versus the ratio of volume to surface area for copper, nickel and gold with various experiments and atomistic simulations [2].

deformation of $2 \mathrm{D}$ hexagonal crystal sheet structure. The non-homogeneous dislocation nucleation of crystal sheet was studied by this methodology, and dislocation nucleation criterion was deduced. The onset of dislocation nucleation and the criterion distribution map were provided. Furthermore, the loading amplitude corresponding to the dislocation nucleation can be determined by comparison between the stress-strain and criterion-strain curves. The results showed that the dislocation nucleation stress has a relation to the crystal sheet orientation angles.

Using MD method, yield strength of copper with twist grain boundary effect was investigated under shear loading. The critical twist angle, which mediates the hardening mechanism of twist grain boundary, can be obtained. When twist angle is lower than critical angle, the yield strength increases with the increase of the twist angle, and the yield form of twist grain boundary is the dislocations nucleation at misfit dislocation network of the boundary and dislocations piling-up in the grain interior. The yield form of twist grain boundary for tensile stress is the partial dislocation nucleation and propagation. The yield form of twist grain boundary for shear stress is proliferation of the boundary. The study of grain boundary density effect on multi-grain thin film under tension was also made [4]. The relationship between the twin boundary spacing and yield strength was obtained. The tensile and compression yielding forms are the dislocation nucleation and propagation from the in-section between free surface and twin boundary. The shear yielding form is the twin boundary motion perpendicular to the direction of shear stress.

Based on the gradient plastic theory of defect energy, an implementation procedure using finite element was illustrated. Considering rectangular and hexagonal form grains, the mechanical properties of multi-grain thin film have been studied. In the simulation process, dislocations cannot slip across the grain boundary. The slip system affects the hardening of the thin film. The dislocations escape from free surface and consequently make the thin film softening. Otherwise, the dislocations, which pile up at the grain boundary, lead to the thin film hardening. In other words, with the increase in the grain boundary density, the hardening influence of thin film becomes stronger. The simulation results reasonably showed that the grain boundary density is linear to the yield strength and hardening rate, respectively.

Some experiments were carried out on water droplets on silicon wafers with microscale regular pillars structures and fresh lotus leaves, which indicate that the multi-scale micro/ nano-structures on a lotus leaf surface are advantageous in forming a stable gas-liquid interface, thus effectively reducing resistance to fluid flow. These encouraging experimental results can direct us to research on the multi-scale numerical model for practical applications [5].

\section{Dislocation dynamic simulation at sub-micron scale}

Plastic flow in crystal at submicron-to-nanometer scales involves many new interesting problems, and is receiving more and more attention of material and mechanics scientists. The plastic deformation of single crystal at micro-scale was investigated by two methods: discrete dislocation method and non-local plasticity method. The first method can describe the evolution of dislocation microstructure, and characterize the details of plastic flow at micro-scale, but the computational cost is quite huge. The second one can give analytical results to explain the intrinsic relation between different physical quantities, but the material parameters need to be decided by the discrete dislocation simulation results and experimental data [6,7].

In the investigation of discrete dislocation plasticity, a unified computational model for discrete dislocation plasticity was developed by directly combining 3D discrete dislocation dynamics (DDD) and finite element method (FEM) [8]. In this methodology, the discrete dislocation plasticity in a finite crystal was solved under a completed continuum mechanics framework. The procedure of variables transferring between two calculation models is illustrated in Figure 2. As an application, the deformation of micro-pillar under uniaxial compression has been predicted. The results showed that mobile dislocation exhaustion hardening is the main mechanism for flow intermittency and stress heterogeneity inside the crystal plays a key role in the observed strain hardening [9]. Uniaxial tension and compression simulations for $\mathrm{Cu}$ single-crystal micro-pillar are carried out by this unified model to investigate the strain rate $\dot{\varepsilon}$ and size effect under uniform loading condition. With the increase of strain rate, the yield stress of single crystal copper increases rapidly and approximately has a linear relationship with $\log \dot{\varepsilon}$.

At the same time the dislocation microstructures change from non-uniform to uniform, and deformation bands were 


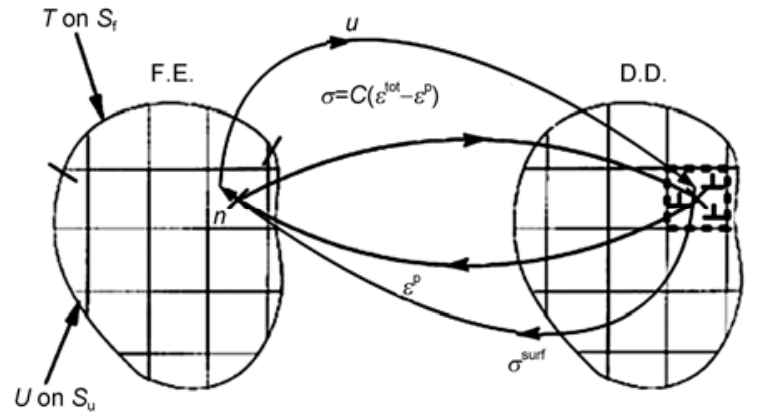

Figure 2 Schematic of variation-tranferring procedure in DDD-FEM methodology [8].

also observed in crystal. The band width increases with the strain rate. And the shear stress in band is higher than that in the neighboring regions. The compression stress-strain curve of $\mathrm{Cu}$ single-crystal micro-pillar at sub-micrometer scale can be divided into three distinct stages: a linear elastic stage, a normal strain hardening stage, and a dislocation starvation induced hardening stage accompanying a rather high stress level. At last, a perfect dislocation-free pillar was obtained in the simulations [10]. The volume averaged compression stress-strain curve of $\mathrm{Cu}$ single-crystal micro-pillar is illustrated in Figure 3, where A, B and C are typical microstructures corresponding to three different stages.

\section{Fracture and damage simulation using XFEM}

Multi-scale analysis approaches can be mainly divided into two groups: information-passing and concurrent. Informationpassing can only transfer a limited amount of variables between different scale models. For engineering practice, concurrent analysis is preferred since it can deal with more complicated coupling behaviors. In concurrent analysis, small domains where critical features such as crack, dislocation and void exist can be locally characterized by molecular model. The rest part where strain gradient is low can be modeled by a coarse scale model like finite element [11]. For the damage evaluation and arbitrary crack growth in the materials, the most natural selection and suitable solving method is the extended finite element method (X-FEM), which can be used to illustrate the weak (damage) and strong (fracture) discontinue problems [12].

The X-FEM is a novel numerical methodology with a great application potential for multi-scale computations and multi-phase coupling problems [13]. An algorithm based on $\mathrm{X}$-FEM was proposed and a program was developed for simulating sub-interfacial crack growth in bi-materials [14]. The maximum circumferential stress criterion and interaction integral were deduced. Numerical analyses of the crack growth in bi-materials give a clear description of the fractural effect exerted by the interface and the loading. Computational results compared with the experiment data, proved that X-FEM is more powerful than the standard FEM in simulating the crack growth path. Further results showed that there is an equilibrium state of mode-I crack in bi-materials if the effects of material inhomogeneity and loading asymmetry counteract each other. Another work related to interfacial crack is using experiment and simulation to determine the mixed mode fracture toughness of the air plasma sprayed (APS) for thermal barrier coatings (TBC)s [15]. The enriched shape function is applied to continuum based (CB) shell element which allows arbitrary crack propagation in curved surfaces, as shown in Figure 4 [16].

\section{Material behavior and constitutive investiga- tions}

For the material behavior investigations, the development of the domain-switching embedded nonlinear electromechanical finite element method is carried out to study the ferroelectric ceramics [17]. Some researchers are focused on developing the plane hybrid stress-function element method for anisotropic materials, which is one of the shape-free
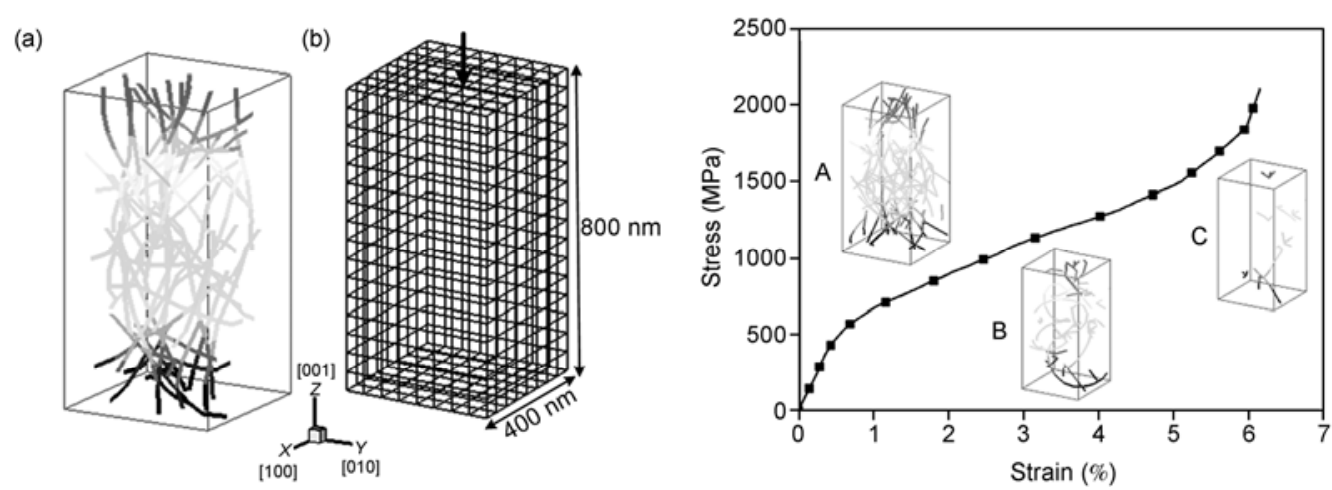

Figure 3 The volume averaged compression stress-strain curve of $\mathrm{Cu}$ single-crystal micro-pillar. (a) The initial annealed dislocation network in the single crystal copper got by an energy minimization process; (b) FE mesh, the constant stress rate is applied on the top surface along [001] direction [10]. 
(a)

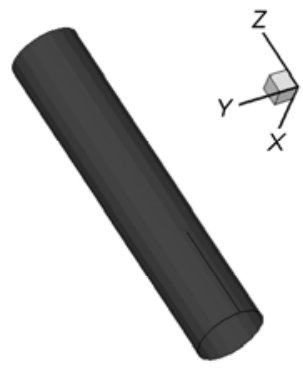

\begin{tabular}{llllllll}
\hline 64.1026 & 128.205 & 192.308 & 256.41 & 320.513 & 384.615 & 448.718
\end{tabular} (c)

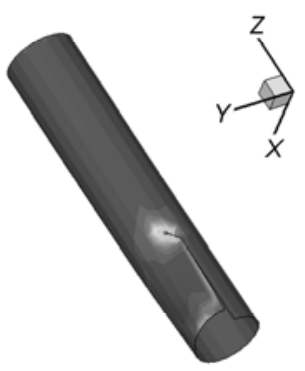

$\begin{array}{lllllll}64.1026 & 128.205 & 192.308 & 256.41 & 320.513 & 384.615 & 448.718\end{array}$

(e)

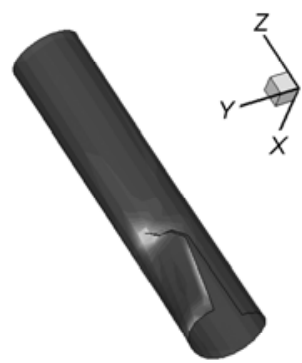

$\begin{array}{llllllll}0 & 64.1026 & 128.205 & 192.308 & 256.41 & 320.513 & 384.615 & 448.718\end{array}$ (b)

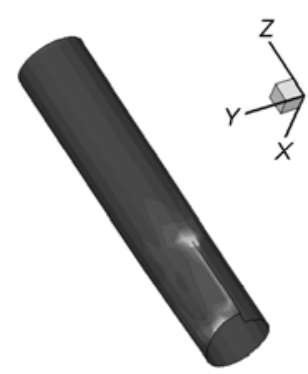

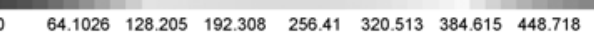

(d)

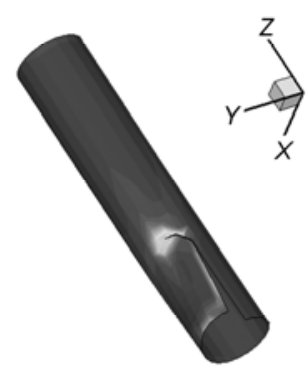

$\begin{array}{lllllll}64.1026 & 128.205 & 192.308 & 256.41 & 320.513 & 384.615 & 448.718\end{array}$

(f)

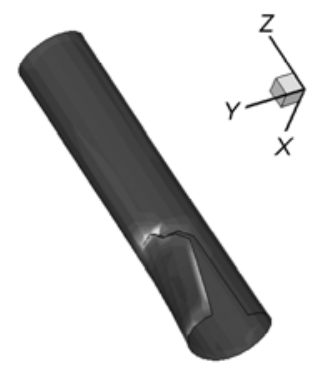

$\begin{array}{lllllll}64.1026 & 128.205 & 192.308 & 256.41 & 320.513 & 384.615 & 448.718\end{array}$

Figure 4 The crack growth in the pipeline [16].

finite element methods [18]. A discontinuous Galerkin finite element approach was obtained for the thermo-elastic coupling problems caused by thermal contact resistance [19]. Due to the absence of long-range order or dislocation-like defects as in crystalline alloys, metallic glasses (MGs) fail by forming intense shear bands which propagate catastrophically without working hardening. The theoretical analysis and the numerical simulation using ABAQUS have been made to evaluate the influence of the initial volume distribution on shear band formation [20]. Viscoelastic materials such as advantaged polymers and their composites are very attractive to damping applications. The damping factor of linear viscoelastic materials was obtained by theoretical studies and simulations [21]. A similar research was conducted to investigate the damping behaviors of the anisotropy tape- spring hinges by means of a theoretical model and simulation [22].

\section{Global-local approach for concurrent multi- scale analysis}

An embedded localization discontinuity method was proposed, which consists of connective elements. The modification of strain field in these elements enabled the jumps in strain associated with the localization band by imposing traction continuity and compatibility within the element [23]. Since then, Belytschko exploited the research field related to enrichment finite element while Fish contributed to the superimposed finite element method [24]. This kind of method is called as s-version FEM. It is proposed to improve the qualities of FEM solutions, compared with the other adaptive FE methods such as h-version or p-version FEM. Apart from adaptive refinement ability, the s-version FEM can be used as a global/local multi-scale computational technique. 
The s-version method uses an independent FE mesh to acquire refined solutions for critical regions of large-scale structures. This separate mesh is overlaid onto the global mesh for the whole structure. These two FE meshes can be of different element size and polynomial order. The consistency of two meshes in displacement field is kept by fixing the boundary of local mesh to be zero. The displacement of the overlapped area equals to the summation of that in the local mesh and in the global mesh. The s-version method has been used to analyze composite plate, micro-macro coupling and fatigue crack growth. A composite plate of fiber-matrix is modeled by global mesh and a local mesh for micro crack. The material properties for global are calculated by mixture rule [25].

Basically, the homogenized material model is used to transit the material properties of the length scale between the micro-scale and the macro-scale. It is feasible to apply for the micro-structures that are periodically arrayed in the structure. And the macroscopic strain field is uniform within the microscopic unit cell domain. The microscopic model is superimposed onto the homogenized model for non-periodical heterogeneous media. Successful achievement on fracture mechanics has attracted many other researchers' attention for s-version FEM. It has been widely used to perform two-dimensional and three-dimensional crack problems. Multiple crack problems have also been presented by modeling each crack as a local mesh as shown in Figure 5 [26]. The reliability of its application on fatigue crack growth problems has been discussed by experimental verification [27]. Figure 6 is the simulation result of the growing path of two interactive cracks. It proves the capability of s-version FEM to capture the arbitrary crack path.

Similar to s-version FEM, a global/local technique called "the Arlequin method" was developed [28]. This approach allows for local modification of an existing coarse model to introduce the micro/local features. One can change the local

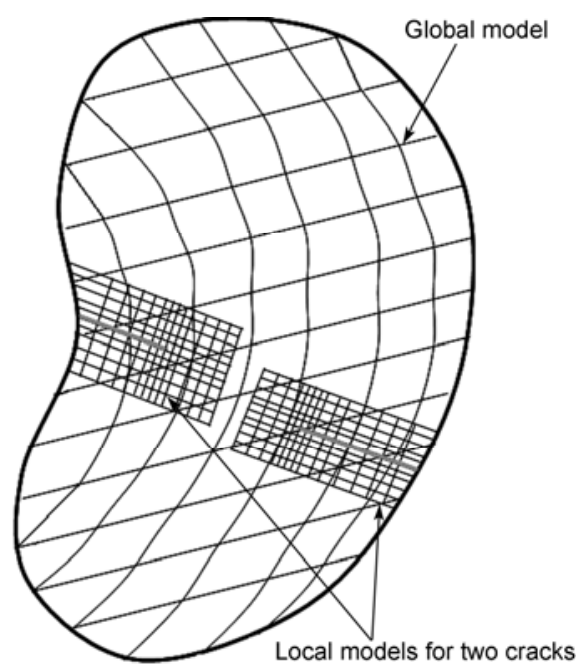

Figure 5 Schematic of the s-version FE method for two cracks problem [26]. behavior law of the global model to study the local damage of the material. Encouragingly, it can transform from continuum mechanics to discrete and more physical mechanics. Different from s-version FEM, the Arlequin method is not the summation of each local domain but the cross of them. It introduced a weight parameter in gluing region to connect the different scale model. The strategy to couple continuum model was studied with molecular model. The gluing zone was called bridging domain. Extension of this study was the application of bridging domain method to dynamic problem [29]. A Lagrange multiplier method was used to ensure the kinematic coherency in the overlapping subregion. The parallel computing performance of this method was also investigated.

\section{The theory and simulation work on aerospace engineering at huge scales}

Operations on large structures and engineering are demanded for the national development, for instance, space missions, nuclear power construction and even the urban atmospheric environment. Yet, special attention should be paid to local problem in large system. The space web structure is a large flexible structure used to capture orbit object protecting the space station from impact. The impact problem of it was analyzed dynamically [30]. The related work is made for the control of large angle maneuvers for the flexible solar sail [31] and optimal four-impulse rendezvous between coplanar elliptical orbits [32]. The wing tip vortex structure behind an airfoil with flaps at the tip of the aircraft has been studied by simulation and experimental work [33]. For the discovering plan on the Moon and to the remote planets, some numerical and theoretical progress has been made related to the design and optimization of a trajectory for Moon departure Near Earth Asteroid exploration [34], and fuel optimal low thrust rendezvous with outer planets via gravity assist [35]. For launching the manned space flight, facilities were also developed for the astronaut mass measurement using linear acceleration method and the effect

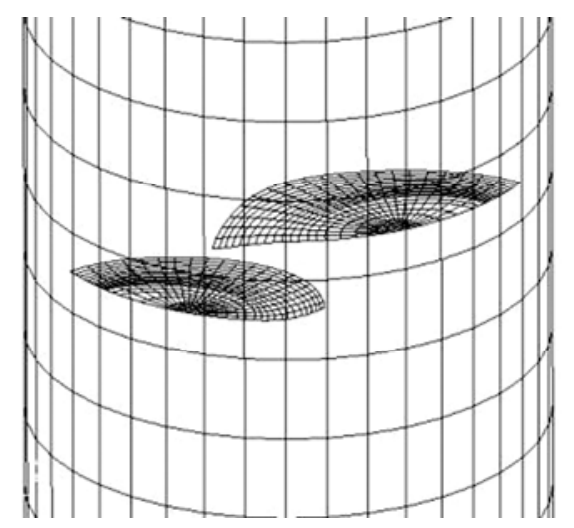

Figure 6 Simulation result of the crack growth path [27]. 
of body non-rigidity [36]. A domain decomposition method was used in the large eddy simulation, in which the urban area was decomposed into a central area, where pollution is the major concern, and a surrounding region, where the pollutant distribution is not important [37]. Therefore, localization problems in a global domain need to be treated by a special simulation methodology in a cost effective way.

1 Liu X M, You X C, Liu Z L, et al. Atomistic simulations of tension properties for bi-crystal copper with twist grain boundary. J Phys D: Appl Phys, 2009, 42: 035404

2 Guo Y, Zhuang Z, Li X Y, et al. An investigation of the combined size and rate effects on the mechanical responses of FCC metals. Int $\mathrm{J}$ Solid Struct, 2007, 44: 1180-1195

3 Liu X M, Liu Z L, You X C, et al. Theoretical strength of FCC single crystal copper based on a continuum model. Chin Phys Lett, 2009, 26: 026103

4 Zhao X C, Zhuang Z, Liu Z L, et al. The study of grain boundary density effect on multi-grain thin film under tension. Comput Mater Sci, 2012, 53: 175-186

5 Hao P F, Yao Z H, Zhang X W, et al. Study of dynamic hydrophobicity of micro-structured hydrophobic surfaces and lotus leaves. Sci China Phys Mech Astron, 2011, 54: 675-682

6 Liu Z L, Liu X M, Zhuang Z, et al. A multi-scale computational model of crystal plasticity at submicron-to-nanometer scales. Int J Plast, 2009, 25: 1436-1455

7 Greer J, Oliver W, Nix W. Size dependence of mechanical properties of gold at the micron scale in the absence of strain gradients. Acta Mater, 2005, 53: 1821

8 Gao Y, Zhuang Z, You X C. A hierarchical dislocation-grain boundary interaction model based on 3D discrete dislocation dynamics and molecular dynamics. Sci China Phys Mech Astron, 2011, 54: 625-632

9 Gao Y, Zhuang Z, Liu Z L, et al. Investigations of pipe-diffusionbased dislocation climb by discrete dislocation dynamics. Int J Plast, 2011, 27: 1055-1071

10 Liu Z L, Liu X M, Zhuang Z, et al. A typical three-stage-hardening mechanical behavior of $\mathrm{Cu}$ single-crystal micro-pillars. Script Mater, 2009, 60: 594-597

11 Fish J. Bridging the scales in nano engineering and science. J Nanopart Res, 2006, 8: 577-594

12 Belytschko T, Black T. Elastic crack growth in finite elements with minimal remeshing. Int J Numer Meth Eng, 1999, 45: 601-620

13 Liao J H, Zhuang Z. ALagrange-multiplier-based XFEM to solve pressure Poisson equations in problems with quasi-static interfaces. Sci China Phys Mech Astron, 2012, 55: 693-705

14 Zhuang Z, Cheng B B. Equilibrium state of mode-I sub-interfacial crack growth in bi-materials. Int J Fract, 2011, 170: 27-36

15 Deng H X, Shi H J, Yu H C, et al. Determination of mixed-mode interfacial fracture toughness for thermal barrier coatings. Sci China Phys Mech Astron, 2011, 54: 618-624

16 Zhuang Z, Cheng B B. A novel enriched CB shell element method for simulating arbitrary crack growth in pipes. Sci China Phys Mech Astron, 2011, 54: 1520-1531

17 Liu B, Fang D N. Domain-switching embedded nonlinear electrome- chanical finite element method for ferroelectric ceramics. Sci China Phys Mech Astron, 2011, 54: 606-617

18 Cen S, Fu X R, Zhou G H, et al. Shape-free finite element method: The plane hybrid stress-function (HS-F) element method for anisotropic materials. Sci China Phys Mech Astron, 2011, 54: 653-665

19 Zheng X P, Liu D H, Liu Y H. Thermoelastic coupling problems caused by thermal contact resistance: A discontinuous Galerkin finite element approach. Sci China Phys Mech Astron, 2011, 54: 666-674

20 Chen Y, Jiang M Q, Dai L H. How does the initial free volume distribution affect shear band formation in metallic glasses? Sci China Phys Mech Astron, 2011, 54: 1488-1494

21 Cao Y P, Ji X Y, Feng X Q. On determination of the damping factor of linear viscoelastic materials using dynamic indentation: A theoretical study. Sci China Phys Mech Astron, 2011, 54: 598-605

22 Yao X F, Ma Y J, Yin Y J, et al. Design theory and dynamic mechanical characterization of the deployable composite tube hinge. Sci China Phys Mech Astron, 2011, 54: 633-639

23 Belytschko T, Fish J, Englemann B E. A finite element with embedded localization zones. Comput Meth Appl Mech Eng, 1988, 70: 59-89

24 Fish J. The s-version of the finite element method. Comput Struct, 1992, 43: 539-547

25 Fish J, Fares N. Micromechanical elastic cracktip stresses in a fibrous composite. Int J Fract, 1993, 60: 135-146

26 Kikuchi M, Wada Y. Interaction effect analysis of two surface cracks using s-version FEM. In: ASME Conference Proceedings, 2009, 48241

27 Maitireyimu M, Kikuchi M. Numerical study of the interaction behavior of two non-coplanar cracks in round bars under cyclic bending. J Solid Mech Mater Eng, 2011, 5: 75-89

28 Dhia H B, Rateau G. Application of the Arlequin method to some structures with defects. Rev Eur Éléments, 2002, 11: 291-304

29 Xiao S, Belytschko T. A bridging domain method for coupling continua with molecular dynamics. Comput Meth Appl Mech Eng, 2004, 193: 1645-1669

30 Yu Y, Baoyin H X, Li J F. Dynamic modelling and analysis of space webs. Sci China Phys Mech Astron, 2011, 54: 783-791

31 Zhang J, Zhai K, Wang T S. Control of large angle maneuvers for the flexible solar sail. Sci China Phys Mech Astron, 2011, 54: 770-776

32 Wang J X, Baoyin H X, Li J F, et al. Optimal four-impulse rendezvous between coplanar elliptical orbits. Sci China Phys Mech Astron, 2011, 54: 792-802

33 Yang K, Xu S J. Wing tip vortex structure behind an airfoil with flaps at the tip. Sci China Phys Mech Astron, 2011, 54: 743-747

34 Chen Y, Baoyin H X, Li J F. Design and optimization of a trajectory for Moon departure near Earth asteroid exploration. Sci China Phys Mech Astron, 2011, 54: 748-755

35 Guo T D, Jiang F H, Baoyin H X, et al. Fuel optimal low thrust rendezvous with outer planets via gravity assist. Sci China Phys Mech Astron, 2011, 54: 756-769

36 Yan $\mathrm{H}, \mathrm{Li} \mathrm{L} \mathrm{M}, \mathrm{Hu} \mathrm{C} \mathrm{H}$, et al. Astronaut mass measurement using linear acceleration method and the effect of body non-rigidity. Sci China Phys Mech Astron, 2011, 54: 777-782

37 Liu Y S, Cui G X, Wang Z S, et al. A composite model for complex building street configuration in a large eddy simulation of local urban atmospheric environment. Sci China Phys Mech Astron, 2011, 54: $716-723$

Open Access This article is distributed under the terms of the Creative Commons Attribution License which permits any use, distribution, and reproduction in any medium, provided the original author(s) and source are credited. 